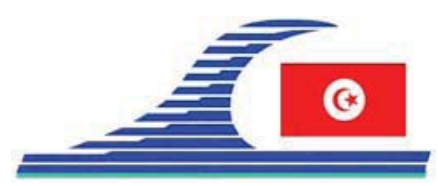

Conférence Méditerranéenne Côtière et Maritime EDITION 1, HAMMAMET, TUNISIE (2009)

Coastal and Maritime Mediterranean Conference

Disponible en ligne - http://www.paralia.fr-Available online

\title{
Méthode de définition d'un point optimum de rejet d'eaux résiduaires en mer : modélisation hydrodynamique et analyse environnementale spatiale multicritères
}

\author{
Fabrice JAVEL ${ }^{1}$, Stéphanie MONERIS ${ }^{1}$, \\ Olivier RAILLARD ${ }^{1}$, Sébastien BOUAT ${ }^{1}$
}

1. SAFEGE, Parc de l'Ile -15/27 rue du Port, 92022 Nanterre, France.

fabrice.javel@safege.fr

\section{Résumé :}

Les rejets d'eaux résiduaires urbaines représentent l'une des principales sources de pollution chronique du littoral méditerranéen. La Communauté Urbaine de Marseille Provence Métropole, la plus importante du littoral méditerranéen français, envisage la possibilité de rejeter au large ces effluents urbains actuellement déversés directement à la côte. Dans le cadre d'une aide à la décision, une méthodologie novatrice, associant modélisation hydrodynamique et analyse spatiale, a été mise au point pour définir le point de rejet optimum et établir le bilan environnemental. Il peut être produit un ensemble d'indicateurs d'effets des différents scénarios de rejet qui, associés à des calculs de ratio coût/bénéfice, peuvent apporter autant d'éléments opérationnels d'orientation des politiques publiques de préservation et restauration de la biodiversité.

\section{Mots-clés :}

Rejets en mer - Turbidité - Sédimentation - Eaux de baignade - Modélisation hydrodynamique - Analyse spatiale - SIG

\section{Introduction}

Les rejets d'eaux résiduaires urbaines représentent l'une des principales sources de pollution chronique des masses d'eaux côtières méditerranéennes. De tels apports, même lorsque les effluents sont traités, impliquent des impacts notamment sur la qualité sanitaire des eaux récréatives et sur les communautés benthiques. Sur les côtes méditerranéennes françaises, le plus important rejet d'eaux résiduaires urbaines est celui de l'agglomération marseillaise (1 865000 équivalent-habitants de capacité). Dans une démarche de réduction des impacts de ces rejets entamée dès 1987, la Communauté Urbaine de Marseille Provence Métropole mène des investigations sur l'opportunité de rejeter les effluents au large par le biais d'un émissaire. Au vu des enjeux financiers d'un tel projet, il était nécessaire d'apporter, dès le stade des études préliminaires, des éléments sérieux quant à son intérêt environnemental. La méthodologie d'analyse présentée ci-après vise ainsi à définir le point de rejet optimum et à établir le bilan du gain environnemental.

DOI: $10.5150 / \mathrm{cmcm} .2009 .053-4$ 


\section{Matériels et méthodes}

\subsection{Modélisation hydrodynamique}

L'outil de modélisation employé, SeaMer_3D, est le résultat de 25 années de développement dans les universités de Brest et Marseille, puis à l'IFREMER (modèle $M A R S$ ) et justifie de très nombreuses applications, ainsi que de la participation à des campagnes de validation et à plusieurs bancs d'essais internationaux (BRETON \& SALOMON, 1996). Ce modèle numérique 3D simule de manière intégrée les courants, la température et la salinité sous l'action de la marée, du vent, de la pression atmosphérique et de la houle. Les conditions aux limites (courant, température et salinité) sont fournies par les sorties du modèle Menor (Ifremer) afin de prendre en compte les phénomènes de méso-échelle. Le module biologique associé permet de représenter finement le comportement en mer des germes bactériens selon leur durée de survie (T90).

\subsection{Protocole expérimental}

Parmi les multiples altérants présents dans les effluents urbains, trois paramètres ont été sélectionnés pour être modélisés par le système: les germes bactériens (impacts sanitaires), ainsi que les matières en suspension et la sédimentation (impacts écologiques). Pour chaque point de rejet étudié (comprenant le rejet actuel à la côte), les simulations sont réalisées selon divers scénarios combinant conditions météoocéaniques et débits de rejet. Des intégrations à l'échelle annuelle sont ensuite réalisées en considérant l'occurrence des conditions météo-océaniques et des débits de rejet.

\subsection{Analyse spatiale}

\subsubsection{Indicateurs d'impact et valeurs seuils}

a) Impacts sanitaires

L'indicateur d'impact sanitaire retenu est le cumul du temps (nombre d'heure sur l'année) durant lequel la concentration en germes bactériens (Escherichia coli, germe témoin de contamination fécale) est supérieure à 1000 UFC / $100 \mathrm{ml}$ (valeur seuil de bonne qualité - AFSSET, 2007). Quatre classes d'impact ont été arbitrairement définies (en pourcentage de l'année) : [0] - [0,1 - 5] - [5 - 20] - [> 20].

b) Impacts sur les communautés benthiques

Concernant les matières en suspension, le manque de données scientifiques de référence et la complexité des phénomènes d'altération associant à la fois les paramètres de durée, de fréquence et de niveau d'exposition, ont conduit au choix d'un indicateur fondé sur une moyenne annuelle sur l'ensemble de la masse d'eau. L'indicateur relatif à la sédimentation est un simple bilan de masse rapportée à une surface de référence. Pour ces deux indicateurs, la démarche d'établissement des seuils s'est fondée dans un 
premier temps sur la définition d'un niveau de référence d'une zone proche et non affectée par les rejets. Les classes d'impact ont ensuite été déterminées selon une analyse comparative et itérative de la zonation effective des impacts connus (voir notamment PERGENT-MARTINI \& PERGENT, 1996) et de celle prédite par les simulations des rejets dans la configuration actuelle («calage» des seuils). Quatre classes d'impact ont été définies allant de « impact non significatif » à « fort impact» :

- $\operatorname{MES}(\mathrm{mg} / \mathrm{l})[<0,015]-[0,015-0,2]-[0,21-2]-[>2]$;

- Dépôt (g - poids sec/m²/an) [<100] - [100 - 400] - [400 - 1000] [>1000].

\subsubsection{Analyse écologique spatiale}

L'analyse écologique spatiale vise à identifier les sites: (1) nouvellement atteints par les rejets au large et à évaluer le niveau d'atteinte; (2) bénéficiant d'une réduction des impacts par la diminution des rejets à la côte et à évaluer le niveau d'amélioration. Cette analyse concerne les impacts sur les usages (baignade et plongée) et les peuplements (herbier à Posidonia oceanica, ceintures à Cystoseira amentacea var stricta, biocénoses de la roche infralittorale et concrétions coralligènes). La série d'opérations nécessaires à l'analyse est menée au sein d'un Système d'Information Géographique (SIG - ArcView v. 9.2, Esri®) : (1) importation dans le SIG des bilans annuels des simulations, interpolation sous forme de raster (méthode des voisins naturels); (2) soustraction entre les données en situation actuelle (rejet à la côte) et en situation envisagée (rejet au large) ; (3) classification selon les seuils définis précédemment ; (4) croisement avec les cartographies des usages et peuplements.

\section{Résultats}

L'analyse décrite précédemment permet de produire un ensemble d'indicateurs d'effet (positif et/ou négatif) des scénarios de rejet basé sur les surfaces de peuplements et le nombre de sites d'usages (baignade et plongée) impactés ainsi que sur les niveaux d'impact. Associée à des calculs de ratio coût/bénéfice cette grille apporte autant d'éléments opérationnels de décision et d'orientation des politiques publiques de préservation et restauration de la biodiversité littorale.

\section{Conclusions}

L'ensemble de la démarche méthodologique associant modélisation hydrodynamique, définition d'indicateurs puis de seuils d'impact et enfin analyse spatiale est susceptible de trouver de nombreuses applications dans les projets de rejets en mer (eaux usées ou ou saumures d'usines de dessalement) ou plus largement les projets impliquant un transfert de polluants (sensu lato) dans les masses d'eau. Des efforts de recherche sont néanmoins encore nécessaires pour mieux comprendre les phénomènes et dynamiques d'impact des rejets chroniques notamment sur les herbiers à Posidonia oceanica. 


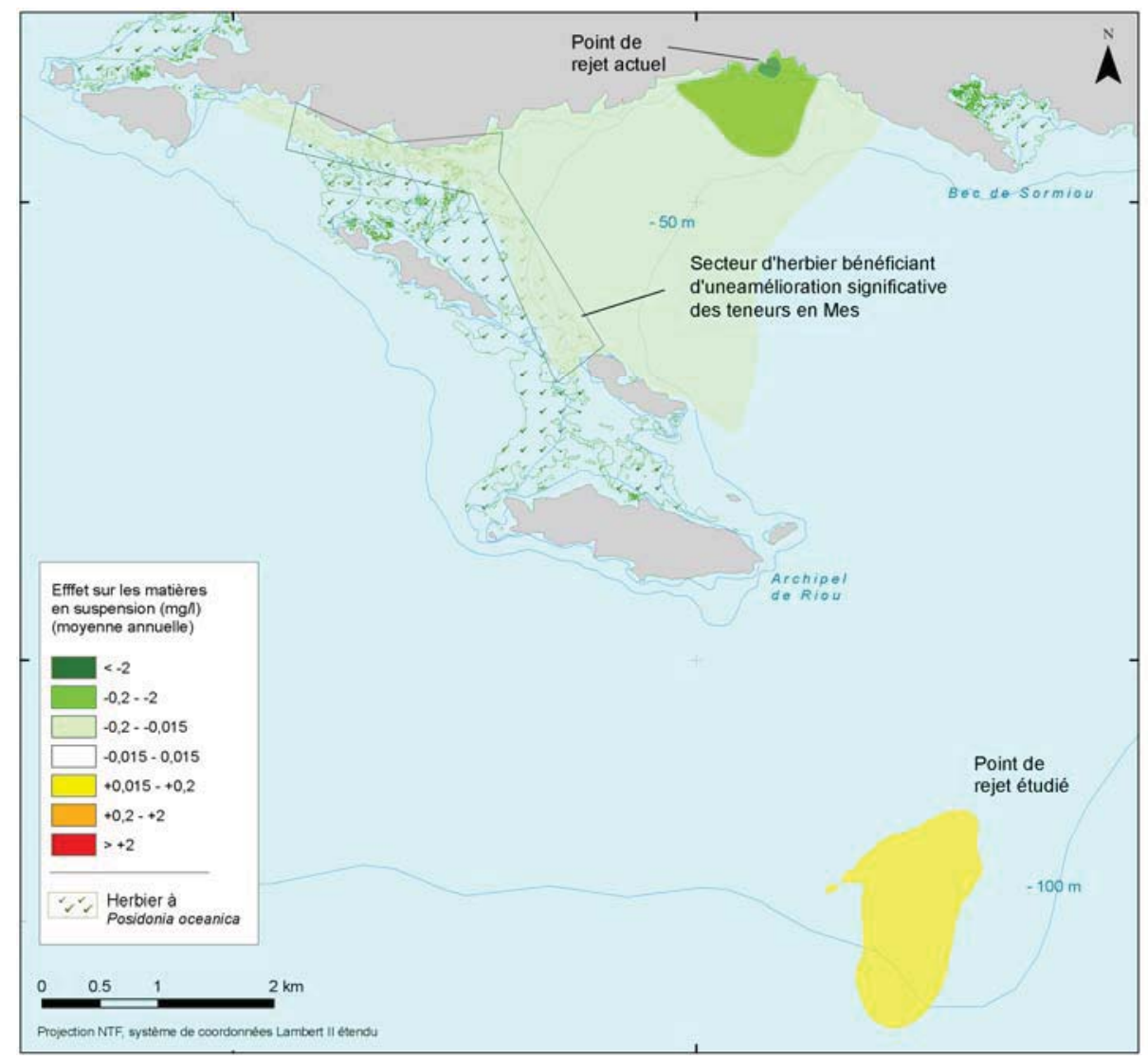

Figure 1. Exemple de superposition entre la cartographie des herbiers à Posidonia oceanica et le zonage d'effet sur les teneurs en matières en suspension.

\section{Références bibliographiques}

AFSSET (2007). Qualité microbiologique des eaux de baignade - : Valeurs seuils échantillon unique pour les eaux de baignade : étude de faisabilité méthodologique. Rapport méthodologique, $55 \mathrm{p}$.

GESAMP (2001). Protecting the oceans from land-based activities - Land-based sources and activities affecting the quality and uses of the marine, coastal and associated freshwater environment. Rep. Stud. Gesamp No. 71. UNEP, Nairobi, 162 p.

BRETON M., SALOMON J.C. (1996). Comparative experience in parallelizing hydrodynamic codes on Sun workstations and Convex 3840. Mmarie annual meeting, Cagliari, 18-19 January, 13 p.

PERGENT-MARTINI C., PERGENT G. (1996). Spatio-temporal dynamics of Posidonia oceanica beds near a sewage outfall (Mediterranean - France). In: KUO J., PHILLIPS R.C., WALKER D.I., KIRKMAN H. (Eds.), Seagrass Biology: Proceedings of an International Workshop, Univ. Western Australia publ., pp 299-306.

SOLTAN D., VERLAQUE M., BOUDOURESQUE C.F., FRANCOUR P. (2001). Changes in Macroalgal Communities in the Vicinity of a Mediterranean Sewage Outfall After the Setting Up of a Treatment Plant. Mar. Poll. Bull., 42(1), pp 59-70. 